Ciência e Natura, Santa Maria v.38 Ed. Especial-

IX Workshop Brasileiro de Micrometeorologia, 2016, p. 477 - 483

Revista do Centro de Ciências Naturais e Exatas - UFSM

ISSN impressa: 0100-8307 ISSN on-line: 2179-460X

\title{
Estudo da produção de energia eólica a partir de medições anemométricas da estação de craíbas/AL e simulações com o modelo WASP
} Study of the wind energy production with measurements wind speed and direction from Craíbas/AL
station and simulation with wasp model

\author{
Thays Paes Oliveira ${ }^{1}$. Rosiberto S. Silva junior ${ }^{2}$, Roberto F. F. Lyra ${ }^{2}$, Sandro Correia Holanda ${ }^{3}$ \\ ${ }^{1}$ Aluna de Graduação, Instituto de Ciências Atmosférica, Universidade Federal de Alagoas, Maceió/AL, Brasil \\ 2Professor Dr., Instituto de Ciências Atmosférica, Universidade Federal de Alagoas, Maceió/AL, Brasil \\ 3Professor Dr., Centro de Ciências Agrárias, Universidade Federal de Alagoas, Maceió/AL, Brasil
}

\begin{abstract}
Resumo
A energia eólica é vista como umas das promissoras na geração de energia elétrica, sendo uma fonte de baixo custo e renovável, tem como benefício à diminuição dos impactos ambientais causados pelas Hidrelétricas. Em conjunto com as redes hidrelétricas, a energia produzida através dos ventos contribuirá com o aumento da capacidade de produção de energia do país. Que a partir de dados de velocidade e direção de vento do município de Craíbas no período correspondente a 2014 - 2015, estimou-se o potencial eólico da região. A ferramenta utilizada no tratamento dos dados levantados foi o Wasp, fazendo simulações de três níveis diferentes de medição, produzindo um parque eólico fictício com aerogeradores potentes. Com o auxílio do modelo WASP estimou-se a distribuição da probabilidade de Weibull e os parâmetros de escala A e forma K. A direção predominante do vento é de Sudeste e a melhor densidade de potência e intensidade do vento aconteceu no nível de $100 \mathrm{~m}$ de altura, cerca de $243 \mathrm{~W} / \mathrm{m}^{2}$. Porém quando avaliado a inclusão do parque eólico fictício, o melhor aproveitamento aconteceu a $100 \mathrm{~m}$ de altura com produção em torno de 73,039 GWh, atribuir essa melhora a maior eficiência do aerogerador utilizado na simulação.
\end{abstract}

Palavras-chave: Estimativa de produção, energia renovável, WASP, Simulações, Usina Hidrelétrica.

\begin{abstract}
Wind energy is seen as one of the promising generation of electricity, as a source of cheap and renewable, is benefit to reduce the environmental impacts of the dam. Along with the hydroelectric networks, the energy produced by the wind will help to increase power generation capacity in the country. That from speed data and direction municipality Wind Craibas in the corresponding period 2014 - 2015, estimated the wind potential of the region. The tool used in the treatment of the collected data was the Wasp, making simulations of three different levels of measurement, producing a fictitious wind farm with powerful wind turbine. With the model, WASP helps estimate the probability distribution of Weibull and scale parameters $A$ and $K$. he predominant wind direction is southeast and the best wind power and intensity density levels took place in $70 \mathrm{~m}$ and $100 \mathrm{~m}$ high, with about $201 \mathrm{~W} / \mathrm{m}^{2}$ and $243 \mathrm{~W} / \mathrm{m}^{2}$ respectively. But when evaluated the inclusion of fictitious wind farm, the best use happened at $100 \mathrm{~m}$ tall with production around $73.039 \mathrm{GWh}$, which can be attributed this improvement to increased efficiency of the wind turbine used in the simulation.
\end{abstract}




\section{Introdução}

Com o grande crescimento da população mundial tem-se a necessidade do aumento da demanda de energia elétrica para o consumo tanto industrial como residencial, porém esse grande aumento na demanda de energia dos últimos anos está provocando a escassez dos recursos naturais e energéticos no mundo inteiro.

Com a busca do aumento do fornecimento de energia várias empresas responsáveis tem a necessidade de aumentar sua área de produção contribuindo com o desequilíbrio ecológico e a desocupação de pessoas da região. Com isso vem à necessidade de exploração de novas fontes energéticas, ou seja, as fontes renováveis que são de baixo custo e não contribui para o impacto ambiental da região.

Hoje uma das fontes alternativa bastante utilizada é a energia eólica, vista na Europa e nos EUA como uma das principais fontes renovável de energia. No Brasil algumas regiões como o Nordeste, estão adotando essa fonte alternativa como meio de ajuda no fornecimento, e supri as necessidades da população em geral. Visto que nos últimos anos a demanda de energia elétrica, está sofrendo alguns problemas com seu fornecimento, como exemplo desse problema, vemos o aumento abusivo do preço da energia em todo o País.

O Brasil é um País tipicamente alimentado por usinas hidroelétricas, que de acordo com o Ministério de Minas e Energia representa cerca de 73,5\% do total nacional em 2005 (MME,2006). Como exemplo de Usina Hidrelétrica tem a Usina de Xingó que pertence ao complexo hidrelétrico do Nordeste instalada no Rio São Francisco, o principal rio do Nordeste Brasileiro possuindo uma área de drenagem de 609.386 $\mathrm{km}^{2}$ e sua bacia hidrográfica cerca de 630.000 $\mathrm{Km}^{2}$, a potência nominal total da Usina de Xingó é de 3.162.000 KW de potência instalada (CHESF).

De acordo com AMARENTE, BROWER, et al., 2001, o Brasil possui um potencial de energia eólico estimado cerca de 143,5 GW no nível de $50 \mathrm{~m}$ de altura, sendo que esse valor pode ser ultrapassado, se haver uma exploração que utilize geradores com mais de $50 \mathrm{~m}$. O nordeste Brasileiro possui em torno de $75 \mathrm{GW}$ sendo este o maior potencial estimando do Brasil, concentrando-se em áreas costeiras.

O objetivo do trabalho consiste em fazer análises dos três níveis de medição da estação de monitoramento de Craíbas, utilizando como ferramenta o modelo atmosférico WASP, determinar qual dos níveis mais se adequa para geração do potencial eólico energético da região e comparar os resultados com a Usina Hidrelétrica de Xingó instalada no Rio São Francisco.

\section{$2 \quad$ Metodologia}

\section{1 Área de Estudo}

A área de estudo está localizada no Estado de Alagoas sendo representada pela estação anemométrica de monitoramento localizada no município de Craíbas (figura 01). O município está nas coordenadas geográficas $9^{\circ} 37^{\prime}$ de latitude sul e de $36^{\circ} 46^{\prime}$ de longitude oeste, com altitude aproximadamente 252 metros. De acordo com o IBGE, possui cerca de 272,847 $\left(\mathrm{Km}^{2}\right)$ de área territorial e uma população estimada 22.641 (IBGE, censo 2010). 


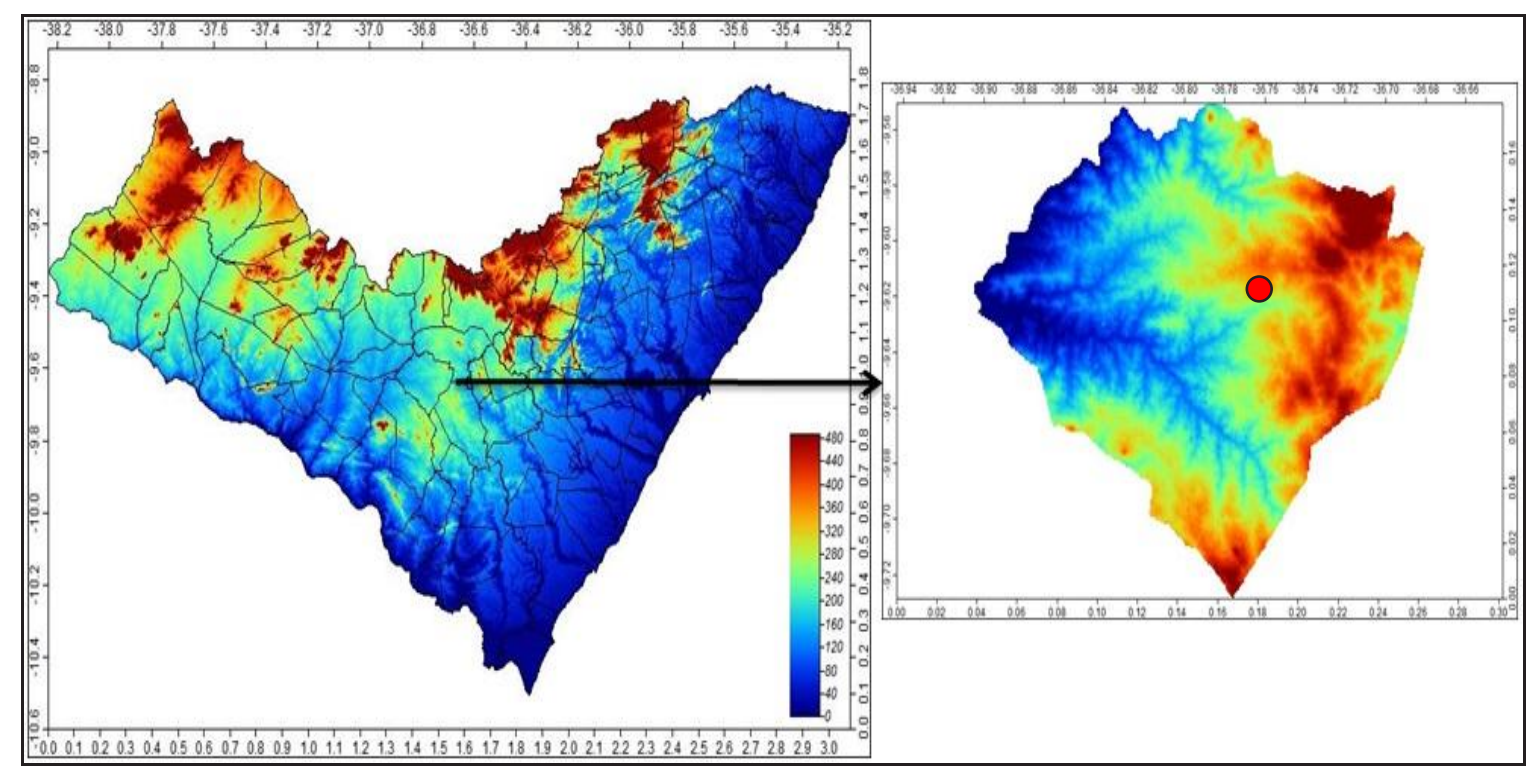

Figura 01: Localização do município de Craíbas/AL, área de estudo

\subsection{Período de estudo}

Os dados de vento utilizados para o estudo estão compreendidos no período correspondente $2014-2015$.

\subsection{Medições}

Utilizou-se dados observados de velocidade e direção do vento, esses dados foram coletados da estação anemométrica de Craíbas.

Dados de três níveis de observação a 50m, $70 \mathrm{~m}$ e $100 \mathrm{~m}$.

O modelo atmosférico WASP foi utilizado para o tratamento de dados e obtenção dos resultados, sendo como o modelo especializado para o mapeamento do potencial eólico.

\subsection{Ferramentas utilizada}

O WASP (Wind Atlas Analysis and Aplication Program) é um modelo numérico que estima o potencial eólico com alta resolução, recomendado para uso em terrenos com topografia de declives baixos ou suavizados. Os parâmetros de fluxo de calor utilizados no modelo são para uma atmosfera levemente estável. Segundo Machado 2008, a velocidade do vento varia com a altura, seguindo aproximadamente uma equação de tipo estática, conhecida como lei da potência.

Seu objetivo principal seria calcular o potencial eólico da região de interesse tomando como base dados de entrada que seriam velocidade e direção do vento, topografia da região visando conhece a rugosidade e a existência de obstáculos, e sendo possível realizar simulações com uma ou várias turbinas as condições reais da região.

Demostrando de forma equacional temos:

$$
V(z)=V\left(Z_{r}\right)\left(\frac{Z}{Z_{r}}\right)^{n}
$$

Onde, 


\section{$V(z)=$ Velocidade na altura $Z$}

$Z=$ Altura acima do solo

$Z_{r}=$ Altura de referencia

$n=$ Coeficiente de extrapolação

Para determina a serie temporal de dados de vento é necessário a utilização da distribuição de probabilidade, a distribuição que mais se adequa para descrever o regime do vento em uma região é a distribuição de Weibull. Tendo seus fatores de escala A ( $\mathrm{m} / \mathrm{s})$ e de forma K (adimensional). Sendo demostrada da seguinte forma:

$$
f(u)=\frac{K}{A}\left(\frac{u}{A}\right)^{K-1} \exp \left(\left(\frac{u}{A}\right)^{K}\right)
$$

Onde,

$f(u)=$ Frequência de ocorrência da velocidade do vento $u$

De acordo com Machado as turbinas instaladas para um parque eólico devem esta separadas a uma distância cerca de 3 a 5 vezes do diâmetro do seu rotor.

Nas imagens seguintes 2, 3 e 4 representadas pela rosa dos ventos, a frequência e a intensidade do vento, que a partir das análises estatísticas do modelo ajustado pela distribuição de Weibull para as alturas de $50 \mathrm{~m}, 70 \mathrm{~m}$ e $100 \mathrm{~m}$. Observa-se que nas 3 imagens a direção do vento são as mesmas sendo predominantes de Nordestes nas respectivas alturas.

$$
\begin{aligned}
& A=\text { Fator de escala } \\
& K=\text { Fator de forma }
\end{aligned}
$$

Os fatores acima relacionados são os parâmetros estatísticos que definem a distribuição dos ventos estando relacionados pelo ajuste da distribuição de Weibull.
O Map editor uma parte do WASP que está separada, fornece para o modelo dados de topografia, nele podem determina altura e a utilização do solo, permitindo a criação do mapa topográfico. O OWC é uma ferramenta também separada do WASP, porém ela desempenha um papel importante que consiste em fazer as análises estatísticas da distribuição da velocidade e direção do vento fornecendo gráficos do comportamento do vento da região em estudo.

\section{Resultados e discursões}

O primeiro passo para avaliar o potencial foi decidir qual aerogeradores a ser utilizado para o estudo. Nesse estudo foi utilizado os aerogeradores do modelo Vesta $\mathrm{V}$ para cada nível, contendo 12 turbinas eólicas para exemplificar a criação de um parque eólico fictício. O modelo Vesta foi escolhido, pois se adequou melhor as situações do vento na região.

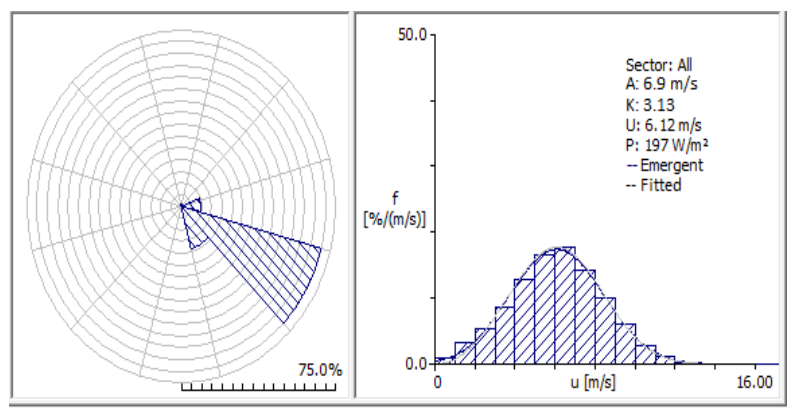

Figura - 2 (a) Direção Predominante do Vento. (b) Frequência e intensidade do vento no nível de 50m: perido 2014 á 2015.

Analisando os níveis de medição notou que em $50 \mathrm{~m}(\mathrm{U}=6.12 \mathrm{~m} / \mathrm{s})$ e sua densidade de potência total $\left(\mathrm{P}=197 \mathrm{~W} / \mathrm{m}^{2}\right)$ como se esperado mostrou a baixo dos demais níveis $70 \mathrm{~m}$ e $100 \mathrm{~m}$, que obteve para $70 \mathrm{~m}(\mathrm{U}=6.21 \mathrm{~m} / \mathrm{s})$ e $(\mathrm{P}=201$ $\left.\mathrm{W} / \mathrm{m}^{2}\right)$ e para $100 \mathrm{~m}$ teve $(\mathrm{U}=6.72 \mathrm{~m} / \mathrm{s})$ e $(\mathrm{P}=243$ $\left.\mathrm{W} / \mathrm{m}^{2}\right)$.

Para o nível 50m utilizou o Vesta V 47 (660/ $200 \mathrm{KW}$ ) - tendo altura do rotor de $47 \mathrm{~m}$, o resultado da simulação do parque eólica gerou 


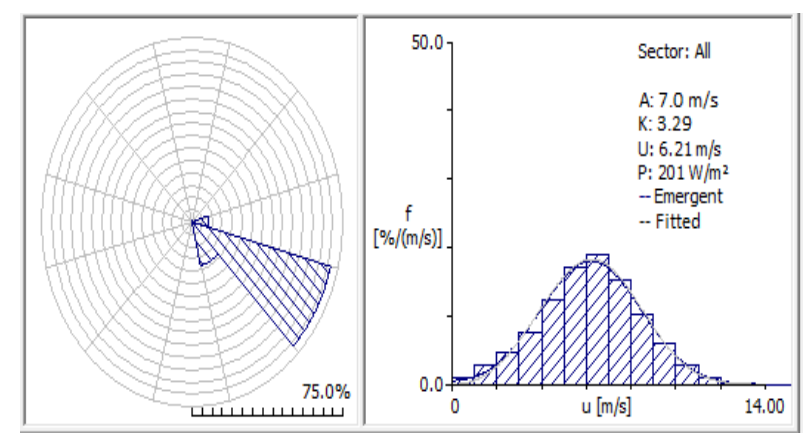

Figura - 3 (a) Direção Predominante do Vento. (b) Frequência e intensidade do vento no nível de 70m: perido 2014 á 2015.

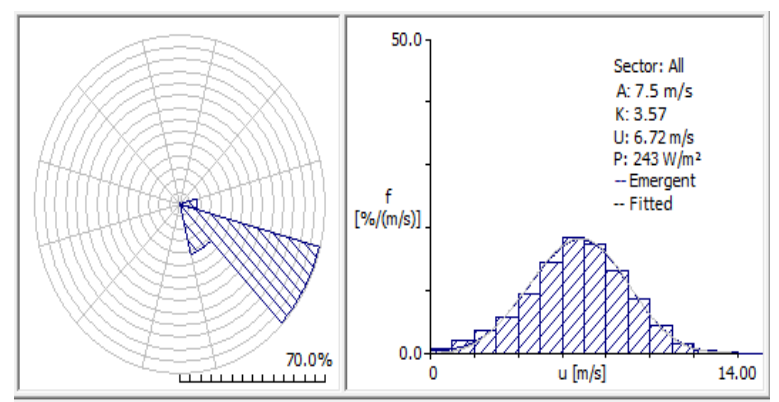

Figura - 4 (a) Direção Predominante do Vento. (b) Frequência e intensidade do vento no nível de 100m: perído 2014 á 2015.

cerca de 17,769 GWh anuais. Em comparação com a Usina Hidrelétrica de Xingó que tem a produção de Energia anual para Alagoas cerca de 20.294 GWh no ano de 2006. O parque eólico fictício representa $0,087 \%$ da produção da usina hidrelétrica de Xingó.

Para o nível de 70m utilizou o Vesta V 661.75 MW (cuja altura do rotor é de $66 \mathrm{~m}$ ), obteve como resultado de simulação do parque eólico o total de 33,331 GWh anualmente.

Em comparação com a Usina Hidrelétrica de Xingó, o parque eólico fictício produziria cerca de $0,16 \%$ (ver tabela 3 ).

Para o parque eólico simulado ao nível de 100m utilizou o aerogerador Vesta 100-02.6 MW (com a altura do Rotor de $100 \mathrm{~m}$ ), sua produção de energia eólica mostrou resultado de 73,039 GWh. Fazendo uma relação com a produção de energia da Usina Hidrelétrica de Xingó, observou-se um percentual de produção equivalente a $0,35 \%$ da produção por parte do parque eólico fictício (ver tabela 2).
Onde, $\mathrm{U}=$ Velocidade Média do Vento, $\mathrm{P}=$ Densidade de Potência Total, K= Fator de Forma, A $=$ Fator de Escala

A tabela 2 mostra ainda um resumo do comportamento do vento no período de estudo e seus níveis de medição. Pode observa tanto em termo de potência como em termo de produção de energia eólica o nível de 100m apresentou

maior estimativa de produção de energia eólica, sendo possível indicar a instalação de aerogeradores de maior porte (com altura do rotor de $100 \mathrm{~m}$ ). Vale destacar que uma análise do custo benefício se faz necessário para avaliar se a diferença de produção de energia entre os níveis seria suficiente para determinar a altura ideal para instalação dos aerogeradores. 
Tabela 2- Comparação dos Níveis de Medição da Estação Anemométrica de Craíbas

\begin{tabular}{c|c|c|c|c|c}
\hline \multicolumn{6}{c}{ Potencial Eólica da Estação Anemométrica de Craíbas } \\
\hline $\begin{array}{c}\text { Nível de } \\
\text { Medição }\end{array}$ & $\mathrm{U}(\mathrm{m} / \mathrm{s})$ & $\mathrm{P}\left(\mathrm{W} / \mathrm{m}^{2}\right)$ & $\mathrm{K}$ & $\mathrm{A}(\mathrm{m} / \mathrm{s})$ & $\begin{array}{c}\text { Energia Produzida } \\
(\mathrm{GWh})\end{array}$ \\
\hline $50 \mathrm{~m}$ & 6.12 & 197 & 3.13 & 6.9 & 17,769 \\
\hline $70 \mathrm{~m}$ & 6.21 & 201 & 3.29 & 7.0 & 33,331 \\
\hline $100 \mathrm{~m}$ & 6.72 & 243 & 3.57 & 7.5 & 73,039 \\
\hline
\end{tabular}

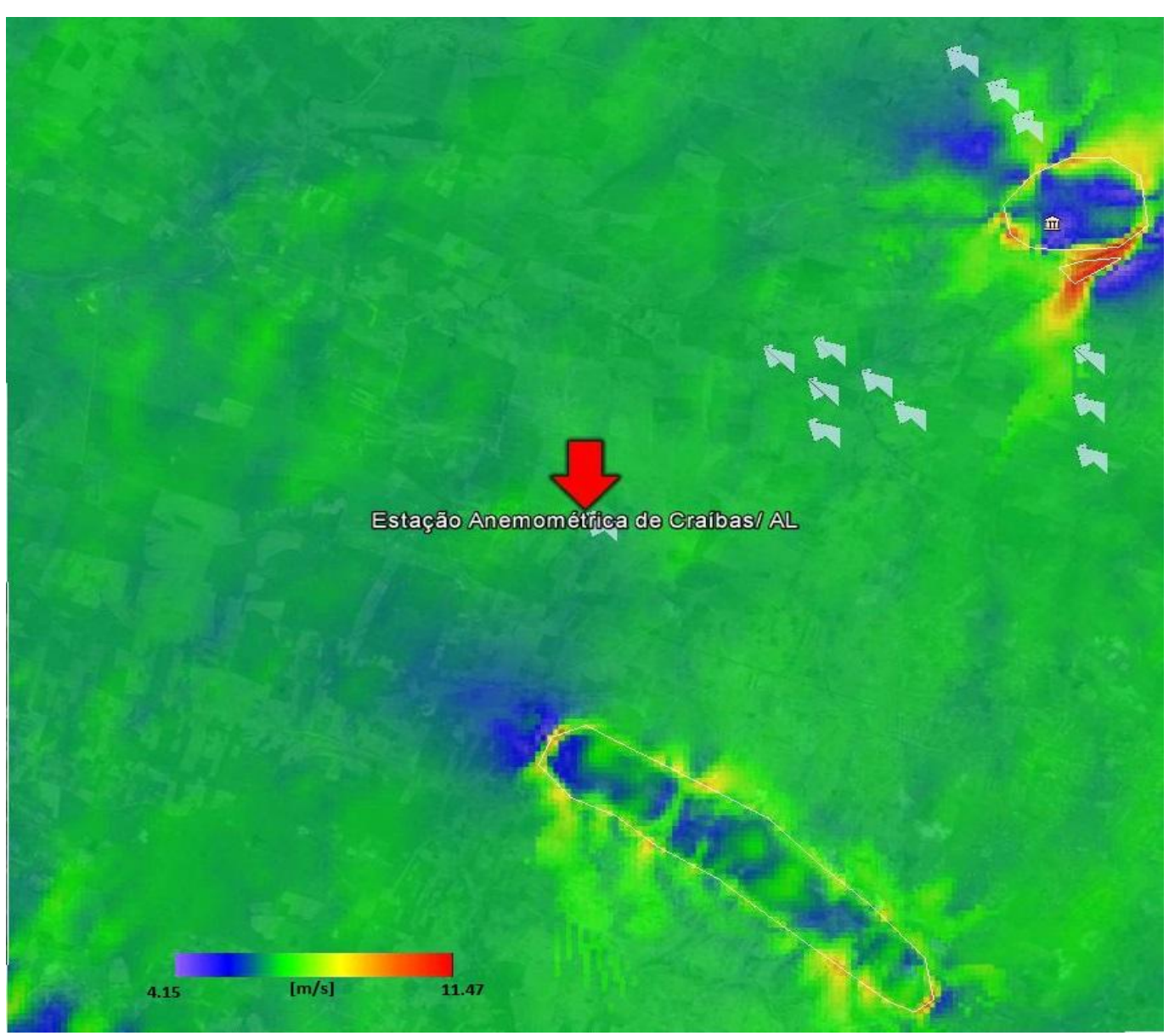

Figura 5- Localização do Parque Eólico Simulado em Craíbas.

A figura 5 é uma ilustração a simulação da instalação de um parque eólico criado com aerogeradores fictícios disponibilizados pelo WASP. Os critérios adotados para a distribuição dos aerogeradores levou em consideração os maiores locais de velocidade do vento, direção predominante do vento, e proximidade da área urbana do município de Craíbas. Na distribuição evitou-se também o efeito de "sombra" entre os aerogerados (altura do rotor multiplicado por 15).

\section{Conclusão}

Em especial a área de estudo apresentou vento predominante de Sudeste e velocidade média do vento entre $612-6,72 \mathrm{~m} / \mathrm{s}$, destaca-se o fato da maior velocidade a $100 \mathrm{~m}$ de altura. Existe uma indicação para instalação de aerogeradores com altura do rotor de $100 \mathrm{~m}$ de altura, quando comparado a produção de energia eólica por níveis.

Em termos de comparação com a produção 
de energia de Xingó. O parque eólico fictício instalado em Craíbas, produziria $0,35 \%$ com aerogeradores de $100 \mathrm{~m}$ de altura, apontando para o ano de 2014 uma produção de 73,039 $\mathrm{GW} / \mathrm{h}$.

\section{Agradecimentos}

A fundação de Amparo à Pesquisa do Estado de Alagoas (FAPEAL) e ao Conselho Nacional de Desenvolvimento Científico e Tecnológico (CNPq) pelo apoio financeiro

\section{Referências}

Amarante, Odilon A., et al. "Atlas do potencial eólico brasileiro." Atlas do potencial eolico brasileiro. Ministerio de Minas e EnergiaEletrobras, 2001.

CALDAS, Danilo Monteiro. "Estudo do Potencial Eólico e Estimativa de Geração de Energia de um Projeto Eólico na cidade do Rio de Janeiro utilizando o WindPro e o WAsP." Monografia de conclusão de Curso (Graduação em Engenharia Elétrica) Engenharia Elétrica da Escola Politécnica, Universidade Federal do Rio de Janeiro, Rio de Janeiro (2010).

CRES Wind Farm. Disponível em: http://www.homepages.ucl.ac.uk/ uceseug/Fluid s2/Wind_Turbines/Blyth_Wind_Farm/Vestas_V6 6.pdf. Acesso em 17 de agosto de 2015.

DE PAIVA NETO, A. C., de Arruda Lyra, A., Chan, C. S., \& Pereira, E. B. Estimativa Preliminar do Potencial Eólico Sobre o Estado de Santa Catarina.

MACHADO, Rogerio Rossi. Estudo do potencial eólico do Pontal do Abreu-Município de Viamão-RS. Diss. Dissertação de Mestrado em Engenharia Oceânica, Rio Grande: Universidade Federal do Rio Grande, 2008.
MARTINS, F. R., R. A. Guarnieri, and E. B. Pereira. "O aproveitamento da energia eólica." Revista Brasileira de Ensino de Física 30.1 (2008): 1304.

RAMOS, DNS. Mapeamento eólico do Estado de Alagoas utilizando ferramentas computacionais e dados observados. 2012. 130 p. Diss. Dissertação (Mestrado em Meteorologia) -Universidade Federal de Alagoas (UFAL), Maceió (AL), 2012.

THE MARITIMES ENERGY ASSOCIATION. Disponível em: http://maritimesenergy.com/flow/uploads/Vestas _V47.pdf. Acesso em 17 de agosto de 2015.

VESTAS. Disponível em: http://www.vestas.com/en/products_and_service s/turbines/v100-2_6_mw\#!technicalspecifications. Acesso em 17 de agosto de 2015.

MME/SPE - MINISTÉRIO DAS MINAS E ENERGIA / SECRETARIA DE PLANEJAMENTO ENERGÉTICO. Grupo de estudos PROCEL/MME (Ceres/MME; Paulo Leonelli/MME; Sergio Bajay/UNICAMP; Jamil Haddad/UNIFEI; Luiz Horta/UNIFEI e PROCEL: George Soares; Eduardo Menandro; Marcos Cordeiro; Maria Cristina Paschoal; Clóvis José da Silva; Marcel Siqueira; Marco Aurélio Moreira; Fernando Perrone; Frederico Castro; Braulio Motta). Em elaboração. Brasília-DF: MME,2006. 